\title{
Anesthésie loco-régionale mandibulaire : \\ l'anatomie au service de la pratique
}

\section{RÉSUMÉ}

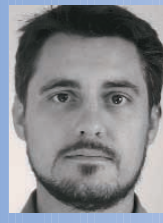

Rémi CURIEN

Faculté d'odontologie de Nancy,

96, avenue du Maréchal de Lattre de Tassigny,

BP 50208

54000 Nancy.

\section{Julien BALLY}

Faculté d'odontologie de Nancy,

Département de chirurgie

et pathologie buccales,

Service d'odontologie,

CHU de Nancy.

\section{Alexandra SOURDOT}

Faculté d'odontologie de Nancy,

Département de chirurgie

et pathologie buccales,

Service d'odontologie,

CHU de Nancy.

\section{Pierre BRAVETTI}

Maître de conférence,

Praticien hospitalier

à la faculté d'odontologie de Nancy,

Doyen de la faculté d'odontologie,

Responsable du département

de chirurgie et pathologie buccale,

Service d'odontologie,

CHU de Nancy.

L'anesthésie loco-régionale au foramen mandibulaire a mauvaise réputation dans la profession étant donné ses fréquents échecs. Ces derniers peuvent cependant être évités par une bonne connaissance de l'anatomie. Nous rappelons ici quelques faits anatomiques classiques mais aussi peu connus, tels que l'existence d'operculations ostéo-fibreuses du foramen mandibulaire. Ces faits appellent une modulation de la technique anesthésique, que nous décrivons.

- anesthésie dentaire

- nerf mandibulaire

- ossification ectopique

- fascia

- ligaments
AOS 2009;248:297-305

DOI: $10.1051 /$ aos/2009404

(C) AEOS / EDP Sciences 


\section{Introduction}

$>$ Dans la pratique de l'anesthésie locorégionale du nerf alvéolaire inférieur dite "à l'épine de Spix», de nombreux échecs sont souvent rapportés, ce qui conduit une partie de la profession à ne plus y recourir et à chercher des alternatives. Cette technique procure pourtant une analgésie de grande qualité et reste encore indispensable dans de nombreuses indications. Or, une connaissance solide de l'anatomie de la région du foramen mandibulaire permet d'en réduire les échecs à un taux quasiment nul. C'est pourquoi quelques rappels anatomiques et leurs implications cliniques pourraient contribuer à redorer le blason de cette technique mal aimée.

\section{Rappels anatomiques}

\section{Situation \\ du foramen mandibulaire}

Le foramen mandibulae (FM), livrant passage au nerf alvéolaire inférieur et à l'artère du même nom, se situe approximativement au centre de la face médiale de la branche de la mandibule (ancien ramus).

Selon Simon et Kömives [1], la variabilité dimensionnelle de la branche mandibulaire est telle qu'il est illusoire d'y définir des repères pour situer le FM. De nombreux auteurs l'ont pourtant tenté. Les résultats des différents travaux consultés sur le sujet sont résumés dans le tableau I. Notons que chez Hayward et al. [7], le FM correspondrait en fait au sulcus retrotoralis (voir infra).

On constate qu'en ce qui concerne la position verticale du FM, les résultats sont extrêmement variables. Toutefois, nous admettrons que le FM est proche du milieu vertical de la branche mandibulaire. Quant à sa position

\section{Tableau II}

Récapitulatif des études portant sur la position du foramen mandibulaire au sein de la branche montante.

\begin{tabular}{lll}
\hline \multicolumn{1}{c}{ Auteurs } & \multicolumn{1}{c}{ Position verticale } & \multicolumn{1}{c}{ Position horizontale } \\
\hline Olivier et Raison [2] & Plus près du bord supérieur du ramus & \\
\hline Paturet [3] & Plus près du bord supérieur du ramus & Milieu du ramus \\
\hline Szolokozy-Syllaba [5] & Milieu du ramus & Milieu du ramus \\
\hline Pelletier [5] & Milieu du ramus & Milieu du ramus \\
\hline Balogh et Csiba [6] & $26 \mathrm{~mm}$ du bord supérieur & \\
& $21 \mathrm{~mm}$ du bord supérieur & $19,7 \mathrm{~mm}$ du bord antérieur \\
& & $11,09 \mathrm{~mm}$ du bord supérieur \\
Hayward et al. [7] & & Troisième quart antéro- \\
& & postérieur
\end{tabular}


horizontale, les avis, là aussi, divergent. Si I'on excepte le travail de Hayward et al. [7] chez qui la définition anatomique du FM est sujette à polémique, on peut considérer d'après la majorité des travaux que le FM est proche du milieu horizontal de la branche mandibulaire.

Selon Olivier et Raison [2], le FM se situerait dans le prolongement du rebord alvéolaire, à $18 \mathrm{~mm}$ en moyenne en arrière de l'alvéole de la troisième molaire (avec des variations tenant à la largeur de la branche mandibulaire qui empêchent la prise en compte de cette alvéole comme repère) et à $30 \mathrm{~mm}$ en moyenne de l'alvéole de la deuxième molaire.

Paturet [3] situe le FM à $2 \mathrm{~cm}$ de la partie moyenne de l'alvéole de la troisième molaire.

Moscovici [8] le situe sur une parallèle au plan occlusal, entre 18,5 à $23,2 \mathrm{~mm}$ du point de Lindsay, point le plus profond de la concavité de la lèvre externe du bord antérieur de la branche montante. Remarquons toutefois que là encore la zone anatomique désignée sous le nom de foramen mandibulaire semble correspondre au sulcus retrotoralis.

Hetson et al. [9] ont tenté de moduler sa situation verticale en fonction de la typologie mandibulaire. D'après leurs résultats, la situation verticale du FM dans le sens inféro-supérieur est corrélée avec l'importance de l'angle goniaque et inversement corrélée avec la largeur antéro-postérieure de la branche mandibulaire. Autrement dit, une mandibule de typologie "rotation antérieure» (branche large et angle goniaque fermé) présentera un FM bas, une mandibule de typologie «rotation postérieure» (branche effilée et angle goniaque ouvert) présentera un FM haut.

\section{Anatomie de la région du foramen mandibulaire}

(fig. 1 et fig. 2 a et b)

Le FM se trouve à l'extrémité antérieure d'une gouttière dirigée en bas et en avant, le sulcus

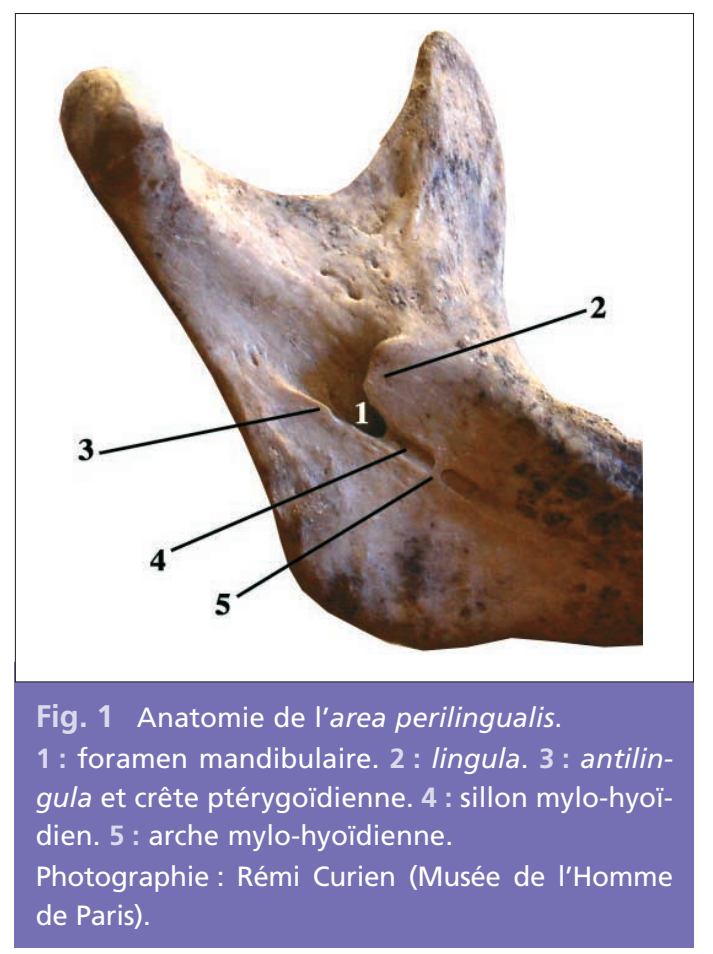


retrotoralis de Lenhossek [10]. II est renforcé par deux berges : une antérieure très prononcée et nettement concave en haut, la lingula mandibulae ou épine de Spix (terme caduc), et une postérieure souvent à peine ébauchée, l'antilingula. Ces deux reliefs sont dédiés à I'insertion des deux faisceaux antérieur et postérieur du ligament sphéno-mandibulaire (LSM) $[11,12]$.

Vers l'avant en bas de la lingula mandibulae, sous la ligne mylo-hyoïdienne, part une gouttière ou un sillon qui s'estompe vers l'avant, le sillon mylo-hyoïdien (sulcus mylohyoideus) parcouru par le nerf mylohyoïdien et l'artère du même nom $[5,11]$.

En arrière du FM se situe la crête ptérygoïdienne, oblique en haut et en arrière, donnant attache à I'aponévrose interptérygoïdienne [13]. Il est difficile de distinguer cette structure de I'antilingula, qui en constitue une simple accentuation.

Juste en dessous de cette crête, sur toute la surface de l'apophyse angulaire, s'observent les reliefs d'insertion du muscle ptérygoïdien médial, dont les fibres partent vers l'avant, le haut et médialement pour s'insérer sur le processus ptérygoïde du sphénoïde. La face latérale de ce muscle et la face médiale de la branche mandibulaire de la mandibule définissent un espace lâche parcouru par les éléments nerveux et vasculaires desservant la mandibule et les muscles manducateurs.
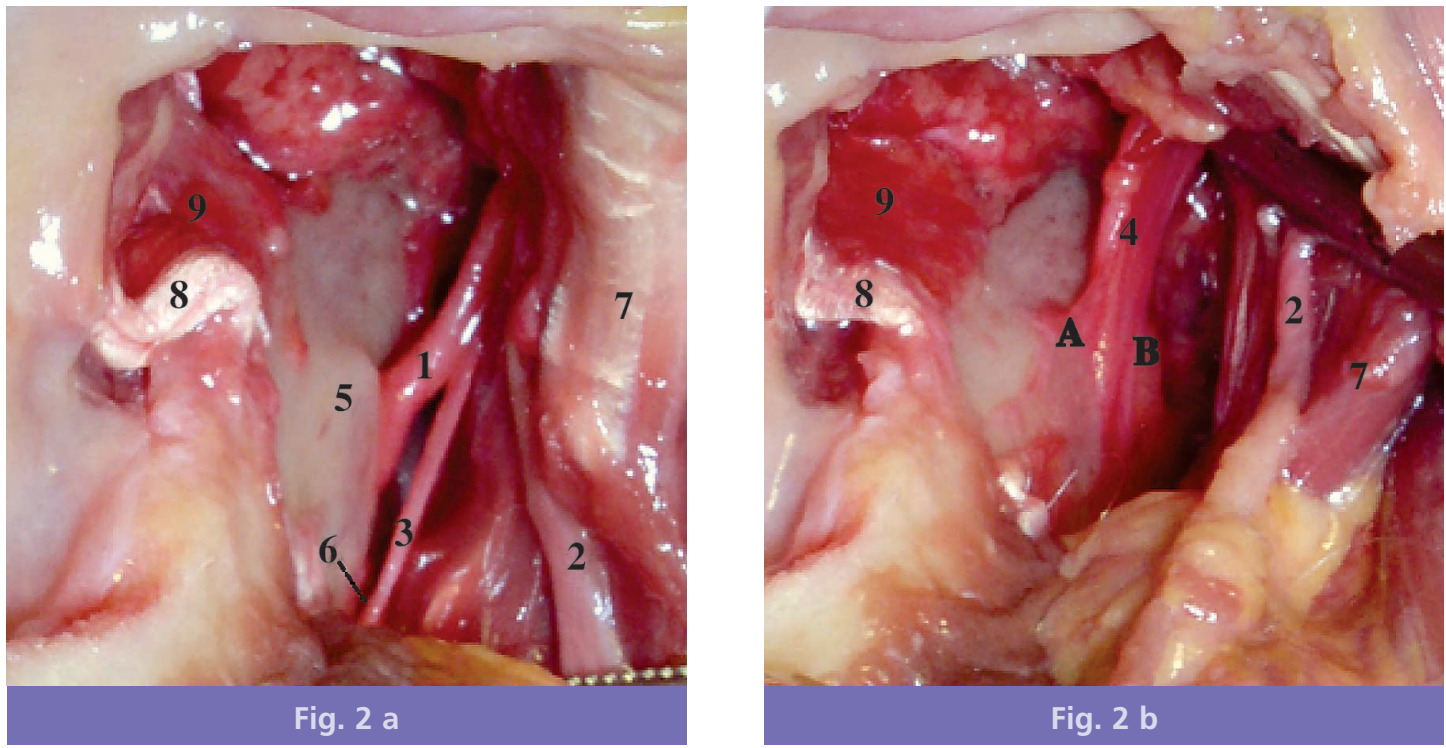

Fig. 2 a et b Dissections de la loge interptérygoïdienne droite en vue antérieure.

a: Sans le ligament sphéno-mandibulaire. b : Ligament sphéno-mandibulaire en place.

1 : nerf alvéolaire inférieur. 2 : nerf lingual. 3 : nerf mylohyö̈dien. 4 : ligament sphéno-mandibulaire. A : faisceau antérieur. B : faisceau postérieur. Entre les deux peut être aisément distinguée la lame vasculaire d'Hovelacque et Virenque. $5:$ lingula. 6 : sillon mylohyoïdien. $7:$ muscle ptérygoïdien interne. 8 : ligament ptérygo-mandibulaire. 9 : muscle temporal.

Photographies : Rémi Curien (Laboratoire d'anatomie de la faculté de médecine de Nancy). 


\section{Système d'operculation du foramen mandibulaire}

(fig. 2 a et b et fig. 3)

Le ligament sphéno-mandibulaire (LSM) est tendu du pourtour du FM à l'épine du sphénoïde. Ce ligament est partie constituante de l'aponévrose interptérygoïdienne, dont il s'individualise néanmoins sous la forme d'une bandelette fibreuse, presque verticale, un peu oblique en bas et en dehors [5].

Vers le bas, il s'étend en s'élargissant pour se fixer au pourtour du FM. Certains auteurs [5, 13] décrivent deux faisceaux, un antérieur et un postérieur, s'insérant respectivement sur la lingula et sur le bord postérieur du FM (ou l'antilingula).

Entre ces deux bandelettes, le LSM émet une sorte de fascia reliant les deux berges du FM ainsi que les bords du sillon mylohyoïdien, transformant le sulcus retrotoralis en canal [12] : il s'agit de ce que Hovelacque et Virenque [14] nomment la «lame vasculaire». Notons que les ouvrages d'anatomie consultés $[2,3,5,11,13]$ ne mentionnent pas cette membrane. Ossenberg [15] confirme pourtant son existence à partir de 36 dissections de la région. Nos propres dissections arrivent à la même conclusion (fig. 2 a et b).

II arrive très fréquemment que ce système aponévrotique s'ossifie au-dessus du sillon mylohyoïdien, créant ce qu'Ossenberg [15] nomme arche mylo-hyoïdienne (voir fig. 1). Dans certains cas, l'arche s'édifie en regard du FM. Périer [16], Ossenberg [15] et Smith [17] décrivent une lamelle osseuse réunissant lingula et antilingula, transformant la gouttière du sulcus retrotoralis en un entonnoir ouvert vers le haut et l'arrière, au fond duquel se trouve le foramen mandibulaire (fig. 3 a à c). Smith [17] nomme cette conformation «forme horizontale-ovale du foramen mandibulaire» (HOFM) et lui attribue une incidence de $3,72 \%$ chez l'homme actuel. Nos propres travaux, portant sur l'observation de 108 branches mandibulaires de mandibules adultes, aboutissent à une incidence de 4,6 \% [Curien R., Étude développementale, morphologique et globale de I'area perilingualis, non publié].
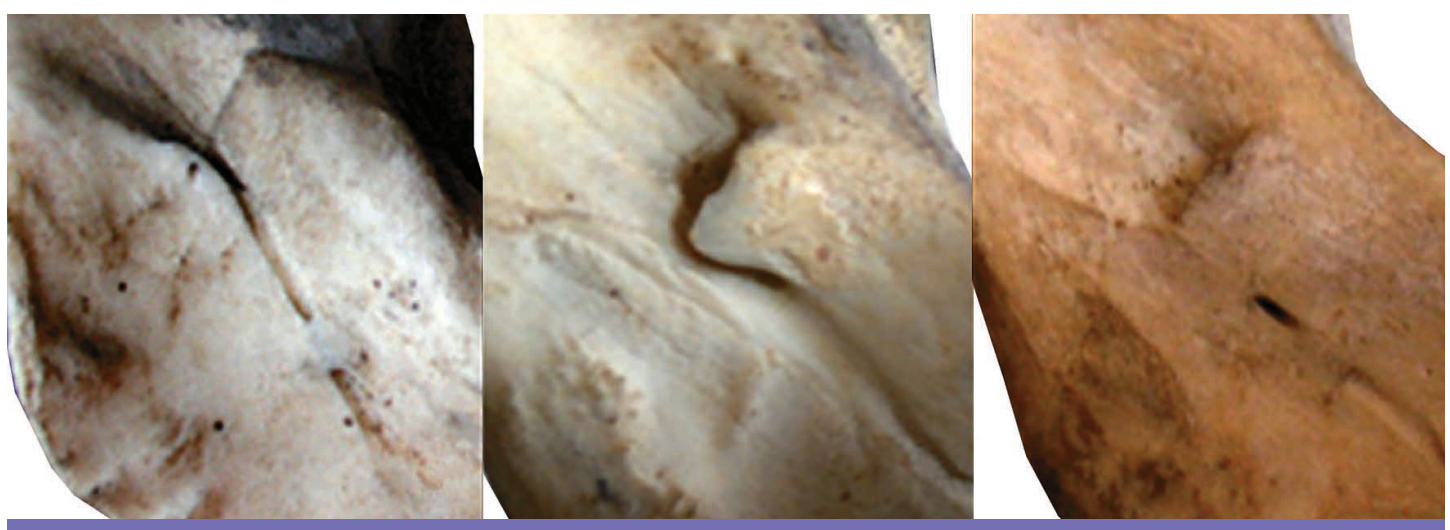

Fig. 3 a à c Aspects de l'operculation osseuse du foramen mandibulaire.

a : forme incomplète par développement lingulaire et antilingulaire. Noter l'association avec une arche mylohyoïdienne.

b : forme incomplète par développement lingulaire prédominant.

c: forme complète où la jonction entre la lingula et l'antilingula est encore visible.

Photographie : Rémi Curien (Musée de I'Homme de Paris et Laboratoire d'anthropologie biologique de la faculté de médecine de Marseille). 


\section{Implications cliniques}

II ressort de ces observations que le foramen mandibulaire se situe au fond d'une sorte d'entonnoir ostéo-fibreux oblique vers le bas et l'avant. L'anesthésie loco-régionale du nerf alvéolaire inférieur aura donc tout simplement pour but d'inonder cette citerne d'anesthésique. L'injection en dehors de cette loge est une cause probable d'échec, étant donné l'existence de cloisonnements aponévrotiques voire osseux empêchant la diffusion de l'anesthésique. Toute la difficulté consiste donc à repérer le passage de l'aiguille dans la citerne. Pour cela, un sondage méthodique (décrit ciaprès) de la zone doit être effectué grâce à l'aiguille d'infiltration. Deux signes tactiles sont à reconnaître : la perception d'une augmentation de la profondeur de sondage latéral der- rière la lingula (passage dans le sulcus retrotoralis) et, au même moment, la sensation du passage au travers de l'aponévrose (s'accompagnant souvent d'une sensibilité accrue chez le patient). Une vision en coupe axiale de la branche mandibulaire au niveau du foramen mandibulaire (fig. 4) permet de mieux visualiser la procédure.

La méthode est la suivante (fig. 4) :

1. Repérage du point de Lindsay à l'aide de l'index de la main droite (ou gauche) et traction des tissus mous en dehors afin de les tendre.

2. Ponction à l'aide d'une aiguille de 40 à 50/100e de diamètre et 30 à $38 \mathrm{~mm}$ de long, juste en dedans de ce point voire légère-

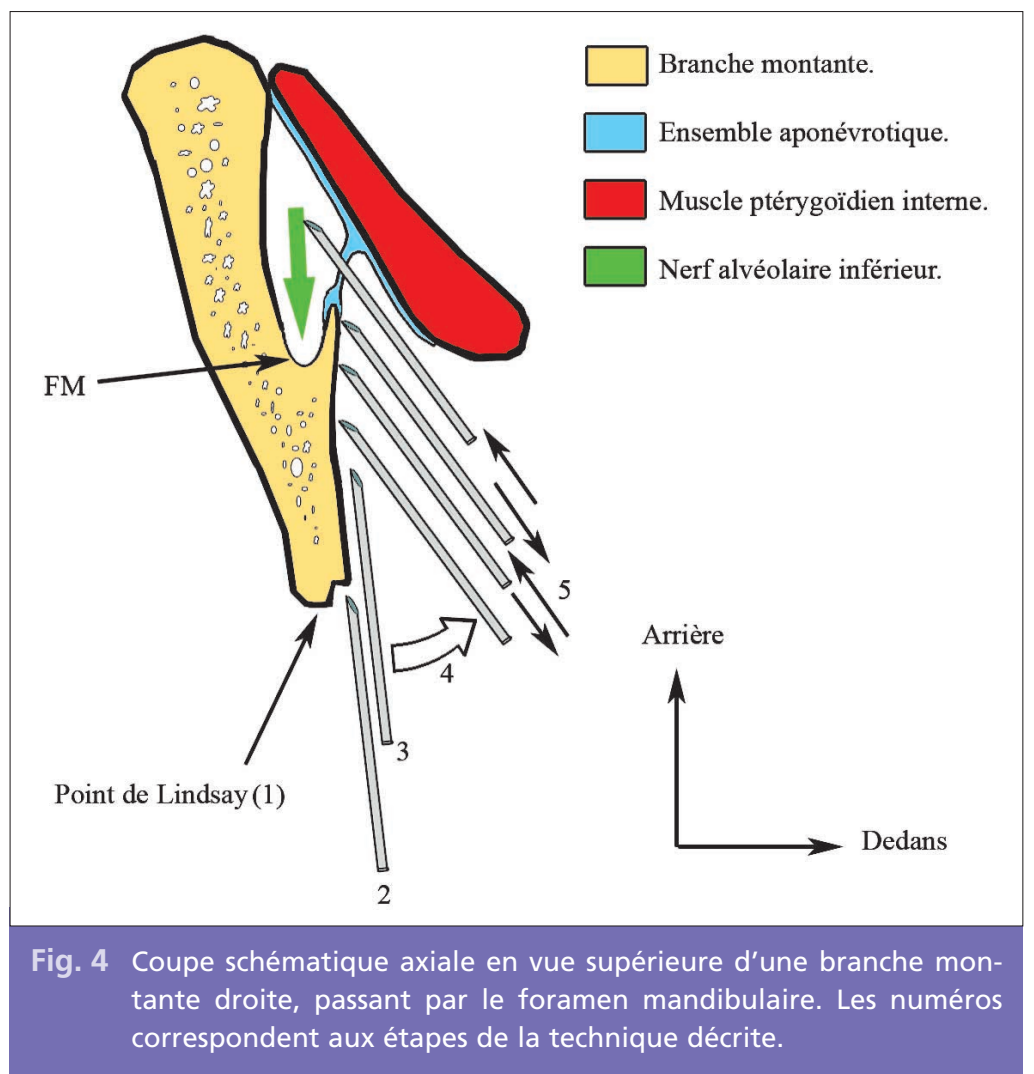


ment plus haut. En effet, l'anatomie montre qu'il est préférable d'injecter dans la zone supérieure du sulcus retrotralis, afin de permettre à l'anesthésique de s'écouler vers cette zone. D'autre part, les études montrent que le FM est souvent haut situé dans la branche mandibulaire.

3. Pénétration de l'aiguille le long de la surface interne de la branche mandibulaire sur environ $1 \mathrm{~cm}$. À cette phase, une butée sur la lèvre interne du bord antérieur de la branche montante est assez fréquente. II suffit alors d'incliner l'extrémité de l'aiguille vers l'intérieur pour passer l'obstacle.

4. Rotation, classiquement décrite, du corps de la seringue jusqu'aux prémolaires de l'hémiarcade opposée. Si ce mouvement n'est pas effectué assez précocement, l'aiguille risque de progresser dans le muscle ptérygoïdien médial, avec risque de douleurs et de contractures postopératoires, et inefficacité de l'injection.

5. Progression vers l'arrière par 1 ou 2 sondages successifs jusqu'à perte du contact osseux et apparition d'un contact ligamentaire à environ $2 \mathrm{~cm}$ du bord antérieur : à ce contact, I'aiguille rencontrera une très légère résistance (différente d'un contact osseux) puis passera en force à travers l'aponévrose.

6. L'extrémité de l'aiguille se trouve alors dans la citerne englobant le nerf alvéolaire inférieur; après test aspiratif, l'injection lente d'une carpule d'anesthésique avec vasoconstricteur au $1 / 200000^{\mathrm{e}}$ peut débuter. Notons qu'à ce moment, il n'est pas rare qu'une aiguille de $30 \mathrm{~mm}$ soit enfoncée jusqu'à la garde dans les tissus mous. Contrairement à la technique classique, il n'est pas nécessaire de rechercher le contact osseux à cette étape. Sa recherche pourrait même s'avérer dangereuse en risquant d'écraser l'artère alvéolaire inférieure contre la surface osseuse et en augmentant ainsi le risque d'injection intravasculaire et/ou d'hématome postopératoire. Rappelons qu'en dehors du contact osseux intempestif, le risque d'injection intravasculaire avec une aiguille de $\mathbf{4 0}$ voire 50/100 est infime : une aiguille de ce diamètre ne fera que bousculer l'artère, mais n'en traversera pas la paroi [18].

Tout au long de la progression de l'aiguille, de légères quantités d'anesthésique devront être successivement injectées afin de diminuer les sensations douloureuses.

Notons qu'à la différence des techniques classiquement décrites [18], où le choix du site d'injection est basé sur la recherche du contact osseux à $2 \mathrm{~cm}$, la technique présente est au contraire basée sur la perte du contact osseux, correspondant à l'entrée dans la zone du FM.

Comme le précisent les données anatomiques, il peut parfois être impossible de traverser la moindre aponévrose, en percevant un contact osseux tout au long du sondage : la présence d'une operculation osseuse du FM est donc à suspecter. Dans ce cas de figure, l'aiguille doit être retirée et la ponction réitérée plus haut, afin de contourner l'obstacle par son bord supérieur.

II n'existe malheureusement aucun signe prédictif de l'operculation osseuse :

- aucun signe morphologique mandibulaire ne permet de la suspecter puisque, selon Smith [17], il ne s'agit pas d'une adaptation fonctionnelle à une certaine robustesse mandibulaire mais un trait génétique isolé ;

- notre propre analyse de 100 radiographies panoramiques numériques (étude réalisée dans le cadre du présent article) montre qu'il est impossible $d^{\prime} y$ individualiser avec certitude la lingula et I'antilingula, et a fortiori une éventuelle operculation osseuse du FM. 


\section{Conclusion}

L'anesthésie loco-régionale au foramen mandibulaire ne doit pas se pratiquer en aveugle, mais en gardant en permanence à l'esprit la conformation anatomique de la zone et en s'aidant de signes simples permettant de s'y repérer. La connaissance des operculations particulières dont la zone fait l'objet, méconnues de la littérature, y est d'un grand secours.

\section{Bibliographie}

1. Simon B, Kömives $\mathrm{O}$. $A z$ allkapocs felhago aganak méreitei. Fogorv Sz 1937;30:49-76.

2. Olivier E, Raison P. Anatomie de la tête et du cou. Paris : Legrand, 1949.

3. Paturet $\mathrm{G}$. Traité d'anatomie humaine. Tome 1 : Ostéologie, arthrologie, myologie. Paris : Masson, 1951.

4. Szolokozy-Syllaba B. Méresi adatok a ligula zsintjének meghatarozasahoz. Fogorv Sz 1953;46:177-80

5. Pelletier M. Anatomie maxillo-faciale. Paris : Maloine, 1969.

6. Balogh K, Csiba A. Les variations anatomo-topographiques de la lingula et de l'orifice du canal dentaire (area perilingualis). Bull Group Int Sci Stomatol 1966;9(2):145-156.

7. Hayward J et al. The mandibular foramen: its anteroposterior position. Oral Surg Oral Med Oral Pathol 1977;44(6): 837-43.
8. Moscovici M. Contribuçào ao estudo do ramo ascendente da mandibula.

Rio de Janeiro.

Thèse : Odontologie :

Rio de Janeiro : 1952.

9. Hetson G, Share J, Frommer J, Kronman JH. Statistical evaluation of the position of the mandibular foramen. Oral Surg Oral Med Oral Pathol 1988;65:32-4.

10. Lenhossek M.

Das innere relief des unterkieferastes. Arch Anthropol 1920; 18:49-59.

11. Rouvière $H$, Delmas $A$ Anatomie humaine descriptive, topographique et fonctionnelle. Tome 1 : tête et cou. $15 \mathrm{e}$ édition. Paris : Masson, 2002

12. Gaspard M. L'appareil manducateur et la manducation. Tome 1 : Anatomie descriptive, ontogenèse et phylogenèse de la mandibule humaine. Paris : J. Prélat, 1978.
13. Laison F, Gaudy JF. Anatomie cranio-faciale. Paris : Masson, 1993.

14. Hovelacque $A$, Virenque $M$. Les formations aponévrotiques de la région ptérygo-maxillaire chez l'homme et chez quelques mammifères. Anat Phys 1913;49:427-488 et 618-699.

15. Ossenberg NS. The mylohyoid bridge: an anomalous derivative of Meckel's cartilage. J Dent Res, 1974;1:77-82.

16. Périer $\mathrm{AL}$.

Contribution à l'étude de la région de l'épine de Spix. Bull. Schweiz. Ges Anthropol Ethnol 1934-1935;9-10.

17. Smith FH.

Evolutionary signifiance of the mandibular foramen area in Neanderthals. Am J Phys Anthropol 1978;48:523-532.

18. Arreto CD, Gaudy JF. Manuel d'analgésie en odontostomatologie. $2^{\mathrm{e}}$ édition. Paris : Masson, 2005. 


\section{SUMMARY}

\section{Mandibular locoregional anesthesia:} anatomy dedicated to practice

Rémi CURIEN,

Julien BALLY,

Alexandra SOURDOT,

Pierre BRAVETTI
Locoregional anesthesia at mandibular foramen, because of frequent failures, has a bad reputation in the dental profession. To avoid these failures, a good knowledge of anatomy is essential. We recall here several anatomical facts, such as osteo-fibrous operculations of the mandibular foramen. These facts lead to a modification of the anesthesic technic, that we describe.

\section{Keywords \\ - dental anesthesia \\ - mandibular nerve \\ - ectopic ossification \\ - fascia \\ - ligaments}

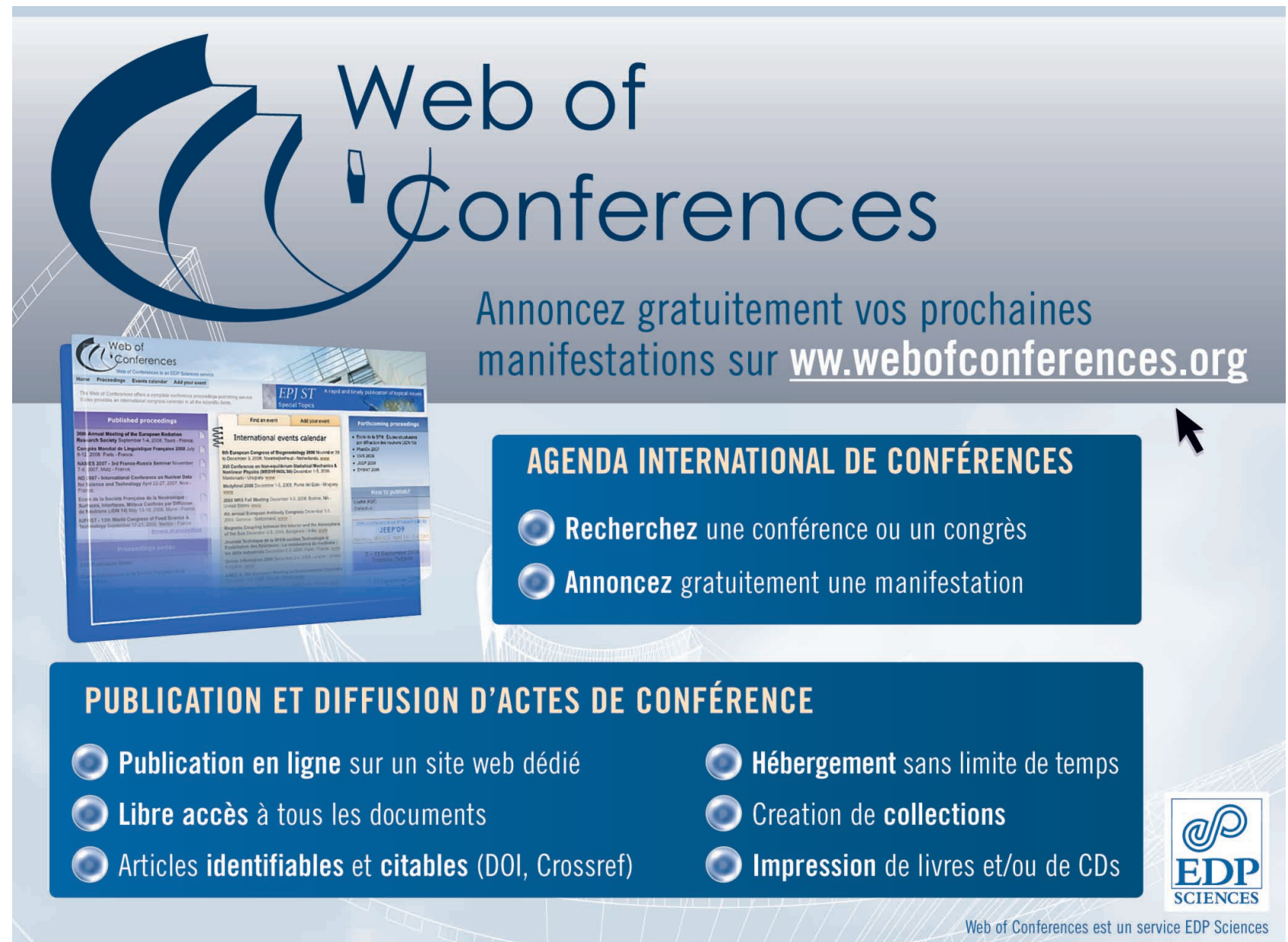

